\title{
TRIPLETS OF GALAXIES: THEIR DYNAMICS, EVOLUTION AND THE ORIGIN OF CHAOS IN THEM
}

\author{
Arthur D. Chernin \\ A. I. Herzen Pedagogical Institute \\ Leningrad, USSR \\ Alexei $V$. Ivanov \\ Institute of Astrophysics \\ Tadjik Academy of Science \\ Dushanbe, USSR
}

1. Recently Karachentsev's group at SAO (6-meter Telescope Observatory) published a list of 84 triple systems of galaxies with their distances, radial (line of sight) velocities, and angular sizes (Karachentseva et al., 1988). This gives a new ground for studies of the dark matter problem which fills the gap between the large cosmic scales (White, 1987; Dekel and Rees, 1987, and Einasto et al., 1977) and the scale of individual galaxies (Erickson et al., 1987). The data on the typical velocity dispersions and linear dimension of the triplets indicate that they contain considerable amounts of dark matter (see also earlier work of Karachentseva et al. (1979). Numerical simulations show that the statistical characteristics of the Karachentsev triplets can be imitated by model ensembles of triple systems with dark matter masses $\mathrm{M}_{\mathrm{d}}=(1-3) \times 10^{12} \mathrm{M}_{\mathrm{O}^{\prime}}$ which is almost ten times greater than the typical mass of stellar galaxies estimated by the standard mass-to-luminosity ratio (Kiseleva and Chernin, 1988). 
Here we report that important information can be drawn from the data on the visible configurations of these systems. The statistics of configurations provide an independent evidence for dark matter in the triplets; moreover, it enables one to argue that dark matter seems to be distributed over the whole volume of the typical triplet forming its commom corona rather than concentrated within individual coronae (or haloes) of the member galaxies.

Let us consider a configuration diagram for triplets (Figure 1) where $A B$ is the largest side of each of the visible configurational triangles; $C B D$ is a part of circle of radius $A B$ with the center in $A ; A B=A D, A C=D C$, and $C D$ is normal to $A B$. Any triangle finds its top (opposite to the largest side) in the area $C B D$, and therefore any triple system can be plotted as a point in this area (Agekian and Anosova, 1967).

Figure 1 presents such a diagram for 46 "physical" triplets of the Karachentsev list. Their distribution over the diagram proves to be fairly homogeneous. The mean number density of the points in each of the four zones (as shown in Figure 1) is the same within the limits $\left|\frac{\delta n}{\langle n\rangle}\right|<1.36 \sigma$,

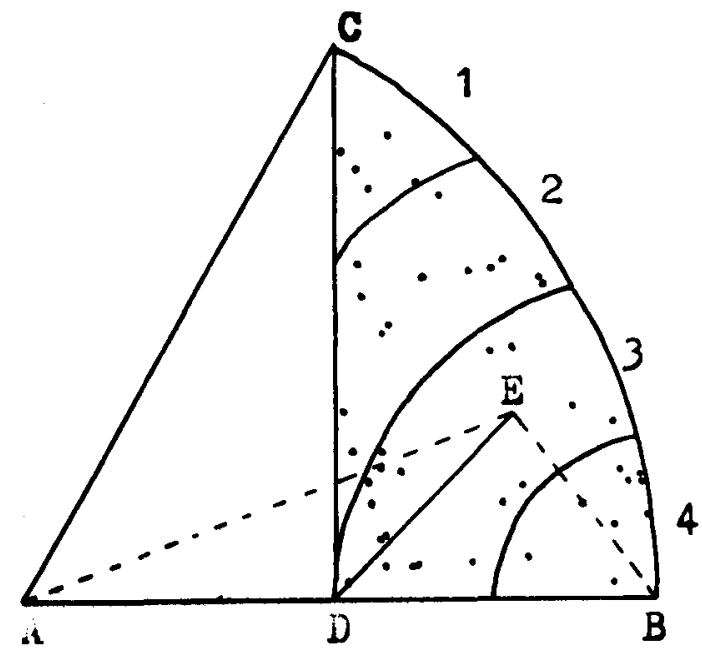
where $\sigma=0.28$, is the r.m.s. deviation in the Monte-Carlo experiment carried out especially for a random scattering of 46 points over the diagram area. 
In our computer simulations, two types of numerical models imitating the observed triplets were studied: isolated three body system with components of equal masses (Model I), and three mass system with distributed dark matter spread spherically over the main volume of the system with density $\rho \sim 1 / r^{2}$ $\left(r_{1}<r<r_{2} ; r_{1}=10 \mathrm{kpc}, r_{2}=1 \mathrm{Mps}\right)$ (Model II). The "softened" potential $\phi(r)=-\mathrm{Gm} /\left(r^{2}+\varepsilon^{2}\right)^{1 / 2}$ for body-body interactions was used, where $\varepsilon=10^{-2}$ in the unit system with $\mathrm{G}=\mathrm{m}=1$. The time was measured in the units of the crossing time $\tau=G\left(\sum_{i} m_{i}\right)^{1 / 2} \sum_{i, j} m_{i} m_{j} /(2|E|)^{3 / 2}$, where $E$ is the total energy of the triplet. A set of ensembles of 46 triplets for each type of model has been compiled and analyzed. The results (Table 1) appear to be in favor of Model II.

TABLE 1

Relative Number Density, $\frac{n-\langle n\rangle}{\langle n\rangle}$, for Configuration Zones $1-4$ of Figure 1

\begin{tabular}{lcccc}
\hline Zones & 1 & 2 & 3 & 4 \\
Observations & -0.32 & 0.10 & -0.12 & 0.34 \\
Model I & -0.70 & -0.80 & -0.30 & 1.80 \\
Model II & -0.26 & -0.24 & 0.16 & 0.34 \\
\hline
\end{tabular}

Any ensemble of Model I reveals a considerable excess of configurations in zone 4: in a typical example shown in $\mathrm{Table}$ 
$1\left|\frac{\delta n}{\langle n\rangle}\right|_{4}=7.3 \sigma . \quad$ Zone 4 contains hierarchial structures in which one side of the configurational triangle is much less than the other two. This excess of configurations in the physical space cannot be smoothed out by projection effects; on the contrary, projection would lead to an even greater excess of this kind in the statistics of visible configurations, as a special analysis shows. Only ensembles of Model II can provide homogeneous distributions of configurations over zones 1 - 4 similar to the observed statistics; in a typical example $\left|\frac{\delta n}{\langle n\rangle}\right|<1.3 \sigma$. Dynamical explanation of such a difference is related to the fact that the probability of formation and existance of a close binary within a system of three gravitating bodies is rather high (Kiseleva and Chernin, 1988). Systems with close binaries look in projection mostly like hierarchical configurations. However, distributed dark matter makes body-body interactions less effective and decreases essentially the probability of binary formation in Model II in comparison to Model I. Table 1 presents an example of ensembles in which this dynamical effect reveals itself most clearly. Generallly, ensembles of Models II demonstrate a wide variety of configuration statistics which merits a special study in the whole context of the statistical properties and stochatic behavior of triple galaxies.

2. Numerical experiments have demonstrated that chaotic dynamics can develop in the triple systems with a characteristic time scale which is sufficiently less than the age of the observed triple galaxies. We studied stochasticity of the 
systems using the method by Casartelli et al. (1976) and computed the maximum Liapunov exponent $\Lambda(t)=\lim _{d(0) \rightarrow 0}\left(\frac{1}{t}\right) \ln \frac{d(t)}{d(0)}$ that gives one the mean exponential rate of divergence of two initially close trajectories. Here $d(t)$ is distance between two trajectories in the phase space. More than 50 individual trajectories are analyzed. The Liapunov exponent proves to be positive for each of them, and reaches the values $201 / \tau$. Figure 2 shows a typical curve for the time dependent Liapunov exponent. It provides a clear indication for dynamical instability in the system.

The numerical experiments enable one to trace also the divergence of trajectories directly. Figure 3 shows a typical evolution of $d(t)$. It demonstrates the high sensitivity of the system to the small difference in the initial conditions which leads inevitably to stochastic behavior of the system in time.

Figure 4 demonstrates the autocorrelation function for the "configuration radius" $R(t)=\left|\frac{D E}{\mid A B}\right|$. The rapid decrease (close to the exponential one) of the autocorrelation function is the characteristic feature of a system with stochastic motions.

The stochastic process described by Figures 2 - 4 develops in an extremely non-monotonic manner. The magnitude of $d(t)$ increases (and then decreases) rapidly within the time period $\lesssim 10$ T. Each of its jumps, by $200-400$ times in value, is due to close approaches of the gravitating bodies. Distributed dark matter forming a common massive corona of the system stimulates transition to chaos because it decreases probability of regular structures like close binaries in these systems. 


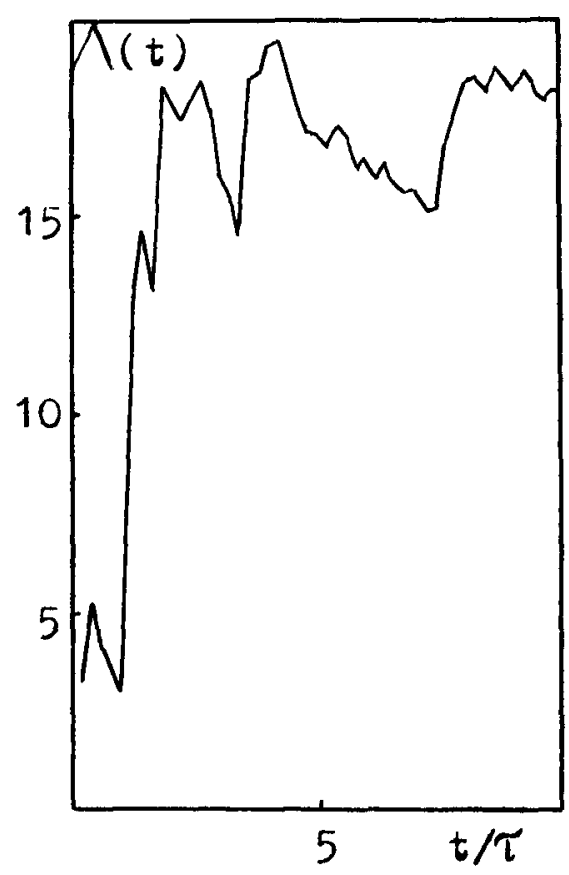

Figure 2

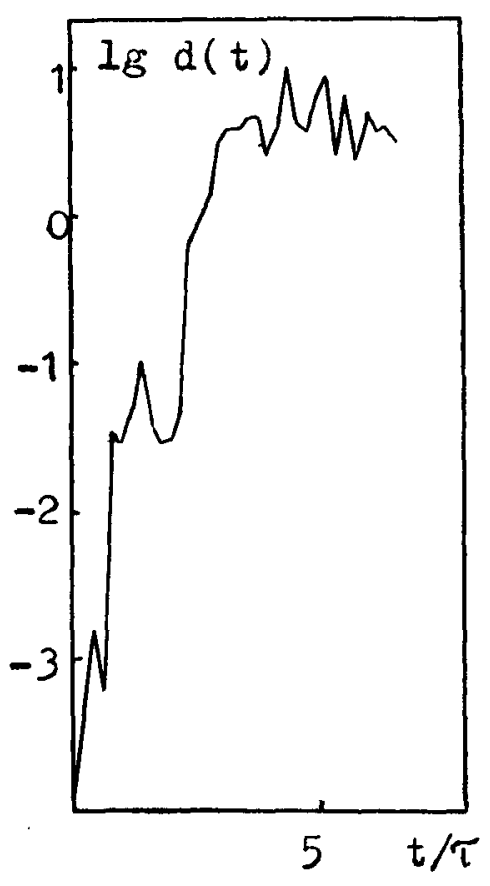

Figure 3

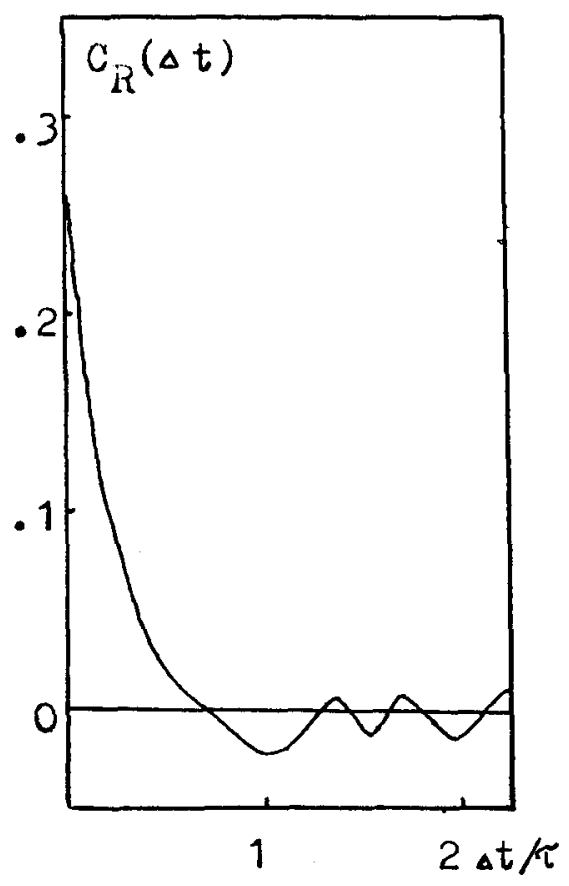

Figure 4 
REFERENCES

Agekian, T. A., and Anosova, J. P., Sov. Astron., 44, 1261 .

Casartelli, M., Diana, E., Galgani, L., and Scotti, A. 1976, Phys. Rev. A, 13, 1921.

Chernin, A., Einasto, J., and Saar, E. 1976, Astrophys. and Space Sci., 39, 53 .

Dekel, A., and Rees, M. J. 1987, Nature, 326, 455.

Einasto, J., Kaasik, A., and Saar, E. 1977, Nature, 250, 309.

Einasto, J., Saar, E., Kaasik, A., nd Chernin, A. D. 1974, Nature, 252, 111 .

Erickson, L. K., Gottesman, S. L., and Hunter J. H., Jr. 1987, Nature, 325,779 .

Karachentsev, V. E., Karachentsev, I. D., and Lebedev, V. S. 1988, Isvestia Spetsial 'noi Astrofizicheskoi Observ., 26, 42 .

Karachentsev, V. E., Karachentsev, I. D., and Sherbanovsky, A. J. 1979, Isvestia Spetsial'noi Astrofizicheskoi Observ., 11,3 . 
Kiseleva, L. G., and Chernin, A. D. 1988, Pis'ma V Astron. Zh., 14,970 .

Ostriker, J. P., Peebles, P.J.E., and Yahil, A. 1974, Astrophys. J. Lett., $193,11$.

White, S.D.M. 1987, Nature, 330, 451. 